\title{
Entropy Correlation Coefficient Technique for Visual Data in Multimedia Sensor Network
}

\author{
Subhash S. \\ M.Tech student \\ MCE,Hassan,Karnataka
}

\author{
Gururaj H. L. \\ Asst. Professor \\ MCE,Hassan,Karnataka
}

\author{
Ramesh B. \\ Professor \\ MCE,Hassan,Karnataka
}

\begin{abstract}
The Wireless Multimedia Sensor Network consists cameras at the sensor node in visual data application. These camera sensor devices capture their observations limited by the FoV as an image. There is a correlation between images captured by multiple cameras at a particular area. This leads to the redundant data transmission in the network. As the sensor node are battery powered and resource limited. Hence these scare resources should be used efficiently. This paper focuses on implementation of entropy correlation coefficient model in WMSN for visual data. The implementation of entropy, joint entropy and mutual information is performed to estimate an ECC which describes correlation characteristics of images observed by camera with overlapped sensing area. SIFT algorithm is used to perform the merging operations between two images. Using the RANSAC algorithm features are matched and the homography between two images are found. The results obtained satisfies the relation between ECC and Joint entropy.
\end{abstract}

\section{General Terms}

Multimedia sensor Network, Visual data

\section{Keywords}

Source image entropy, mutual Information, joint entropy, ECC, SIFT.

\section{INTRODUCTION}

A developing pattern for the recovery of video streams, still pictures and detecting data from the encompassing environment is the WMSN. A WMSN has extensive variety of uses, for example, video reconnaissance, natural checking, and modern procedure control. Contrasted with the scalar information from remote sensor organizes, the sight and sound sensor system ought to transmit their information with certain nature of administration. The vitality utilization, transmission capacity and restricted handling abilities existing with sensor hubs are critical parameters to be considered. Along these lines WMSN requires more mind boggling information pressure strategy for lessening the utilization of vitality and data transmission by the sensor hub. Among the WMSN information, visual data is the most commanding part. Camera gives picture which is connected straightforwardly to the field of perspective that is constrained by detecting bearing. A video breaking down strategy in light of connection is proposed in [4], yet this is utilized for two sensors which don't vary little. Likewise this technique restrains the quantity of sensors to two. Along these lines the preparing of more than two sensors in WMSN for visual data is the issue. In this setting, the paper displays the usage of relationship qualities of pictures secured by the cameras. In view of the connection attributes of pictures the picture mosaicing procedure is done to expel the repetitive information among the pictures furthermore give picture pressure.

Single camera conveyed in a field can cover just a restricted perception which is reliant on its perspective. In the field if the required application needs the range to be watched and to the sink more noteworthy than that of single camera FoV, then there exists lost data in the transmission. As the whole field is of vast when contrasted with the single camera field of perspective, Multiple cameras are joined in the WMSN field for visual data recovery. Consequently the Wireless Multimedia Sensor Network contains substantial number cameras, sent in a field is as appeared in Figure 1.1. It can be seen that there are few chosen range which are application subordinate. With the characterized range of interest cameras covering the region are chosen to send their perceptions to the sink.

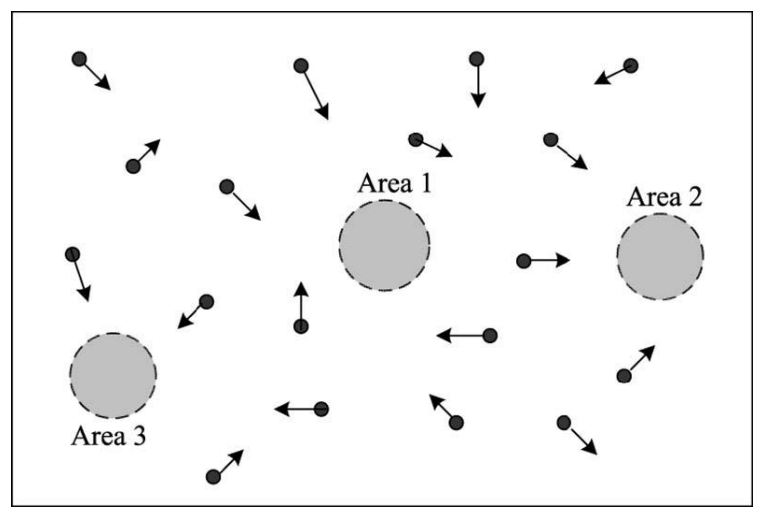

Fig 1.1: WMSN with cameras and areas of interest

Camera sensor hub perceptions are subject to their field of perspective. The Field of View is spoken to as a part. Figure 1.2(a) demonstrates the camera FoV in which camera point is signified as P. The detecting sweep is spoken to as V. $\alpha$ is the balanced point between the detecting course and a sweep of the part. Cameras can mention its objective fact inside its Field of View as it were.

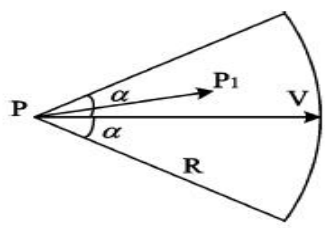

Fig 1.2: (a) camera Field of View 


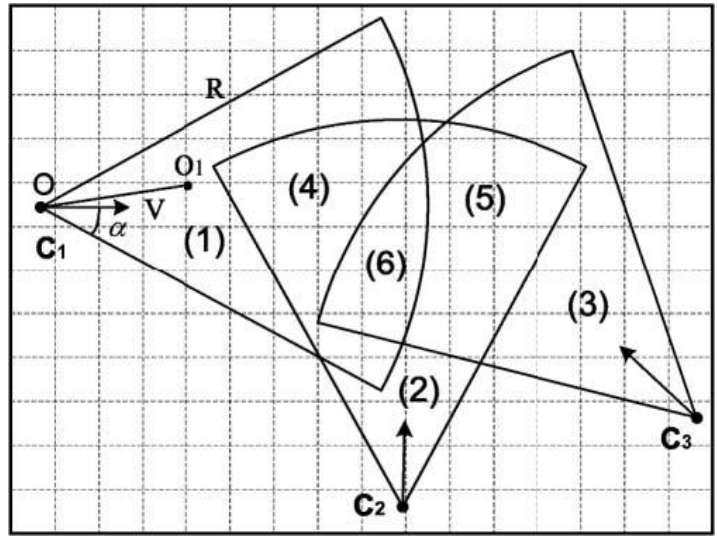

Fig 1.2: (b) Overlapped Field of View

In the field even with range indicated and selecting just the cameras which cover that territory, connection between's camera exists. This is on the grounds that the contiguous camera hubs as a rule shows abnormal amounts of relationship. This outcomes in information repetition in the visual data picked up by the system. Camera detects a 3-D scene and ventures it as 2-D picture. The covered FoV for three cameras is as appeared in Figure 1.2(b). It can be watched that all the three cameras are related to each other. The perceptions made by cameras with covered FoV results a covered picture. This construes there exists relationship between's the pictures secured by the cameras with covered FoV's. This relationship between's pictures speaks to the measure of excess between the watched pictures from the camera.

In this manner with a specific end goal to dispense with the excess in picture SIFT based picture mosaic procedure is connected. At the point when the quantity of pictures are two this strategy can be connected in a straightforwardness way. Trouble emerges when number of pictures to be combined are more than two. Additionally the case with the succession known SIFT can be over and again connected to combine the pictures. Be that as it may, when the arrangement is obscure and this gets to be difficult. Hence to choose which pictures to be utilized for blending procedure is gotten from the Entropy Correlation Coefficient Model. In this manner the out happen to the task gives the answer for excess expulsion for the instance of various pictures, acquired from a WMSN camera perceptions.

\section{RELATED WORK}

Cameras used in the WMSN for visual Information provide their observations in the form of a 2-D image. The processing at the cluster head for the removal of redundancy in the images is performed. As the process is carried out on mages the basics of image processing has to be dealt. The combination of the sensor network and image processing field is implemented in this dissertation. In this direction in the first phase of the dissertation a vast review of literatures available in the area of Wireless Multimedia Sensor Network and image processing applied to the medical images has been conducted.

$\mathrm{Pu}$ wang et al. [2] have proposed a data theoretic picture pressure model with a goal to boost the general pressure of the visual information gathered in a WMSN. The work comprises an entropy-based disparity measure (EDM) plan to gauge the effectiveness of pressure for the joint coding on the pictures gathered by spatially connected cameras. This plan consider just the camera arrangement as inputs without requiring properties of genuine pictures. The contemplations are exceedingly identified with the broadband research center arranged usage. Henceforth the hypothetical ideas from this work on the spatial connection of cameras were received for the study on relationship attributes of cameras.
Rui Dai et al.[1] have proposed a hypothesis of spatial connection model for visual information in WMSNs. With the assistance of camera detecting technique and its usage, a spatial relationship capacity is acquired to depict the connection qualities of visual information saw by cameras with covered field of perspectives. In this paper the Joint impact of numerous associated cameras and an entropy-based structure to gauge the measure of visual information gave by various cameras in the system is produced by relationship show the camera determination calculation is likewise planned.

Devarajan et al.[5] have proposed appropriated calculation for camera adjustment with the programmed, external,metric alignment of a system of cameras with no brought together processor. With enthusiasm for computation of connection taking into account cameras,estimation of every cameras central length, area and detecting bearing in a system is an unquestionable requirement. Henceforth the study on the inside and outside parameter is removed from this paper.

$\mathrm{Wu}$ et al.[?] have proposed a community oriented picture coding and transmission plan to minimize the exertion for information transmission. In this paper Size coordinating technique to coarsely enlist pictures for discovering greatest cover to misuse the spatial connection between's pictures obtained from close-by sensors is introduced. A lightweight and effective foundation subtraction strategy is utilized to recognize focuses in this proposition. Just the areas of target and their spatial areas are transmitted to the checking focus.

From this paper study on Spatial and temporal correlation is performed.

Wiegand et al.[6] have proposed an a diagram of the specialized characters of H.264/AVC, Based on investigation of video coding innovation is done taking into account this paper.

Wu et.al [7] have proposed a strategy for effective pressure and transmission of pictures in an asset obliged multinodes remote system. This can be accomplished by dispersing the workload of packing a picture over numerous adjoining sensor gadgets. Thusly, these arrangements don't investigate the connection of the watched pictures among contiguous sensors. This paper gives study on the adjoining hubs and multihop correspondence in WMSN's.

Wang et.al [8] have utilized auxiliary comparability as an option persuading technique for the outline of picture quality measures. In this paper the customary methodology for picture quality appraisal taking into account affectability of blunder was actualized to demonstrate its constraints. Fundamental ideas on picture quality measure was contemplated from this paper.

Puri et al.[9] have proposed the standards of lossy appropriated pressure from multiuser data hypothesis for PRISM which is a video coding. Crystal empowers exchange of the computationally costly video encoder movement look module to the video decoder. In this plan conveyed video coding was proposed to misuse the adjoining outline relationship. However, the precision was not get for connection strategy among ensuing video outlines, it prompts constrained encoding proficiency of circulated video coding. The idea of casing contiguous was contemplated to contrast it and the camera hub contiguousness.

Pluim et al.[10] have proposed an a depiction of entropy and shared data for the application in medicinal field. The paper introduces the historical backdrop of Entropy,Joint Histogram and shared data. With the history introduced, the paper portrays the use of these nuts and bolts for picture enrollment based shared data for therapeutic pictures. The idea of 
common data, Entropy and joint Entropy connected for picture is utilized to for the usage of individual source Entropy,Mutual data and Joint Entropy of camera pictures.

Studholme et al.[11] have created robotized 3D multimethodology medicinal picture arrangement. This technique is simply rely on upon entropy. In this application where misalignment can be substantial concerning the imaged field of perspective, invariance to cover measurements is an imperative thought. Current entropy measures are investigated and a standardized measure is proposed which is essentially the proportion of the whole of the peripheral entropies and the joint entropy. This paper gives the data about the standardized connection coefficient termed likewise as Entropy Correlation Coefficient. This Entropy Correlation Coefficient shapes the root work of this exposition.

Manjunath et al.[?] have proposed the utilization of Gabor wavelet highlights for surface investigation and give a thorough trial assessment. Correlations with other multidetermination surface characters utilizing the Brodatz composition database show that the Gabor characters give the best example recovery precision. This paper essentially bargains on the picture preparing strategy and specifically utilizing surface data for skimming and recovery of huge picture information. The thought for usage of highlight based identification for the undertaking is separated from this paper.

Jain [12] has proposed an alternate calculations for grouping. Various leveled grouping depicted in this paper as minlinkage or full-linkage or normal linkage is examined. The relationship coefficient between one gathering and another gathering can be gotten. The various leveled bunching plan demonstrates the consolidated pictures as a representation the grouping structure. In the undertaking to acquire the relationship between's on picture and the other various leveled bunching is performed.

A.Annis Fathimaa et al.[13] have proposed an altered type of SIFT calculation for picture sewing. This strategy is actualized utilizing consistent minutes consolidated with SIFT charecteristics is exhibited to decrease the time and computational many-sided quality. It is watched that lone a little part of the nearby view pictures are covered. Consequently, It points in identifying covering part to extract coordinating focuses. The covering locales are resolved utilizing inclination based prevailing edge extraction and invariant minutes. In the concluded area, the SIFT (Shift Invariant Feature Transform) characters are separated to decide the coordinating elements. The enrollment is continued with RANSAC (Random Sample Consensus) calculation and last yield mosaic is gotten by distorting the pictures. The proposed approach results in lessened time and computational

at the point when contrasted with existing techniques. From this paper the SIFT based picture mosaic is extricated to actualize for the undertaking.

In light of the above audit of written works, this thesis expects to study and execute ECC model in WMSN for visual data. The execution of Entropy, Mutual data and Joint Entropy is gotten from technique proposed in [10].Implementation of connection coefficient utilizing the acquired entropy and shared data is as given by [11]. Contingent upon the ECC esteem two picture combining process needs to happen. This usage utilizes SIFT highlight extraction strategy as given from [13]. The joined work upto this stage is introduced in the calculation from [1]. The camera choice in view of the connection coefficient is executed as given by the calculation in [1].

\section{METHODOLOGY}

This section deals about the significance of Joint Entropy and ECC in in WMSN for Visual information. If $\mathrm{Yi}$ is the covered image transmitted to the sink by a camera sensor $\mathrm{Si}$, then the amount of information gained at the sink is $\mathrm{H}(\mathrm{Yi})$. If there is a group of sensor $\mathrm{S}=\mathrm{S} 1 ; \mathrm{S} 2 ; ; \mathrm{SN}$ and their transmitted images are $\mathrm{Y} 1 ; \mathrm{Y} 2 ; ; \mathrm{YN}$ to the sink, then the amount of information gained at the sink will be the joint entropy $\mathrm{H}(\mathrm{Y} 1 ; \mathrm{Y} 2 ;$; YN). Thus the joint entropy of the images has to be estimated.

Entropy correlation co-efficient model for excess expulsion in WMSN for visual data comprises of the taking after stages.

1. Execution of Entropy, Mutual Information and Joint Entropy connected for two source pictures.

a) The Shannon source entropy model is actualized for the picture source entropy estimation.

b) Mutual data as an element of the individual entropy and joint entropy is figured. The idea of joint histogram is utilized to locate the contingent likelihood of the pixel levels in two pictures.

c) Joint entropy is evaluated as an element of individual picture entropy and shared data.

2. Execution of Entropy relationship co-proficient as an element of individual picture source Entropy and Mutual Information got for two source pictures.

3. Usage of picture mosaic in light of SIFT highlight calculation is actualized.

The procedure includes the accompanying strides:

a) Implementation of SIFT highlight calculation to separate the Local elements of the two picture.

b) Implementation to coordinate comparable elements between two pictures.

c) Implementation of Homography framework utilizing RANSAC calculation.

d) Based on the homography framework got play out the required change what's more, wrap the two pictures.

4. Execution of Mutual Information and Joint Entropy connected to numerous source pictures which results as a network.

a) Implement the joint entropy and shared data of all $\mathrm{N}$ pictures taking two pictures at once and structure the qualities in the shared data grid and joint entropy network which are of size [NXN].

b) comparatively process an ECC grid of size [NXN].

c) Merge two pictures in view of the most connected worth got by ECC framework.

This lessens the estimation of $\mathrm{N}$ as $\mathrm{N}-1$.

d) Repeat the strides 12 and 3 for N-1 cycles with the new $\mathrm{N}$ esteem came about because of step 4 .

5. Execution of connection based camera choice calculation.

a) Implement the joint entropy and shared data of all $\mathrm{N}$ pictures taking two pictures at once and structure the qualities in the common data network and joint entropy lattice which are of size [NXN].

b) Also register an ECC lattice of size [NXN]. 
c) Merge two pictures taking into account the slightest corresponded esteem got by ECC framework.

This decreases the estimation of $\mathrm{N}$ as $\mathrm{N}-1$.

d) Decrement the estimation of M.

e) Repeat the progressions from 1 to 4 till the estimation of $\mathrm{M}$ is 1. For reiteration of steps the new estimation of $\mathrm{N}$ and $\mathrm{M}$ must be considered.

\subsection{Joint Entropy for two images}

Consider that two camera sensors A and B cover the required area of interest in WMSN. Let each camera captures one image of it field of view, denoted as image A and image B. The measure in the amount of information obtained when a single source is considered (image A from camera A or image B from camera B) in the form of individual entropy will be less when compared to the joint entropy of both the images $\mathrm{A}$ and B (both camera observations considered). If the complete seen covered by both the cameras have to be sent to the sink then the joint Entropy of the two images is sent rather than individual entropies. Hence, the joint entropy is more likely to be transmitted instead of single source. For the estimation of joint entropy between two images A and B in WMSN (2.14) is used.

\subsection{Entropy correlation coefficient(ECC)}

The entropy correlation coefficient (ECC) provides the correlation degree between two images A and $\mathrm{B}$. The value of ECC ranges from zero to unit. When the source A and B are diffrent the value is zero and when the ECC value is unit it indicates that source are same. The increase in the ECC value implies that the two sources are more correlated. The amount of information obtained together by the two images i.e., the Joint Entropy of two images depends on the ECC. If the correlation degree is more between the two images $\mathrm{A}$ and $\mathrm{B}$, the joint entropy gained from the two images $\mathrm{A}$ and $\mathrm{B}$ is less.

\subsection{Implementation}

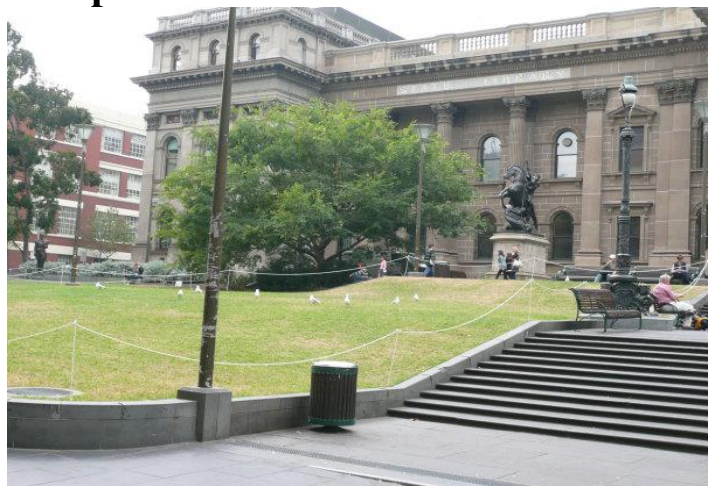

Figure 3.1 (a) Image A

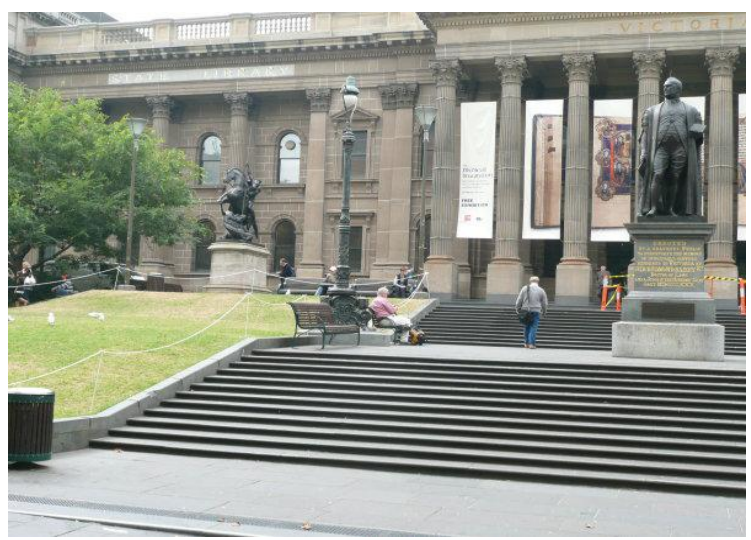

Figure 3.1 (b) Image B
International Journal of Computer Applications (0975 - 8887)

Volume 148 - No.2, August 2016

Table 3.1: Simulation results for two overlapped input images

\begin{tabular}{|l|c|}
\hline Figure & Result from the simulation \\
\hline $\begin{array}{l}\text { Individual source Entropy for } \\
\text { Image A, H(A) }\end{array}$ & 7.5607 \\
\hline $\begin{array}{l}\text { Individual source Entropy for } \\
\text { Image B, H(B) }\end{array}$ & \multicolumn{2}{|c|}{0.2002} \\
\hline $\begin{array}{l}\text { Mutual information between } \\
\text { image A and image B, I(A,B) }\end{array}$ & 0.2002 \\
\hline $\begin{array}{l}\text { Mutual information between } \\
\text { image B and image A, I(B,A) }\end{array}$ & 15:0832 \\
\hline $\begin{array}{l}\text { Joint entropy matrix, H(A,B) } \\
\text { JE =7:5607 }\end{array}$ \\
\hline $\begin{array}{l}\text { Entropy correlation co- } \\
\text { efficient (ECC) matrix }\end{array}$ & ECC = 1 \\
\hline
\end{tabular}

\subsection{Image Feature Detection \\ SIFT ALGORITHM}

SIFT feature detection consists of four main stages.

Stage 1: Scale-space extrema detection

This stage computes all scales and image locations. By using Difference of- Gaussian function it can be implemented efficiently. which identifies the potential interest points that are invariant to orientation and scale.

\section{Stage 2:. Keypoint Identification}

At each estimated location, a detailed model is fit to determine scale and location. Keypoints are selected on basis of measures of their stability.

\section{Stage 3: Orientation assignment}

Based on local image gradient directions multiple orientations are allocated to each keypoints.. All further operations are performed on image information that has been transformed relative to the assigned scale, orientation, and location for each characters, thereby providing constant values to these transformations

\section{Stage 4: keypoint descriptors development}

After identification of keypoints, based on scale and orientation, image gradients are measured. These gradients are transformed into a representation which admits significant levels of local change in illumination and shape distortion. 


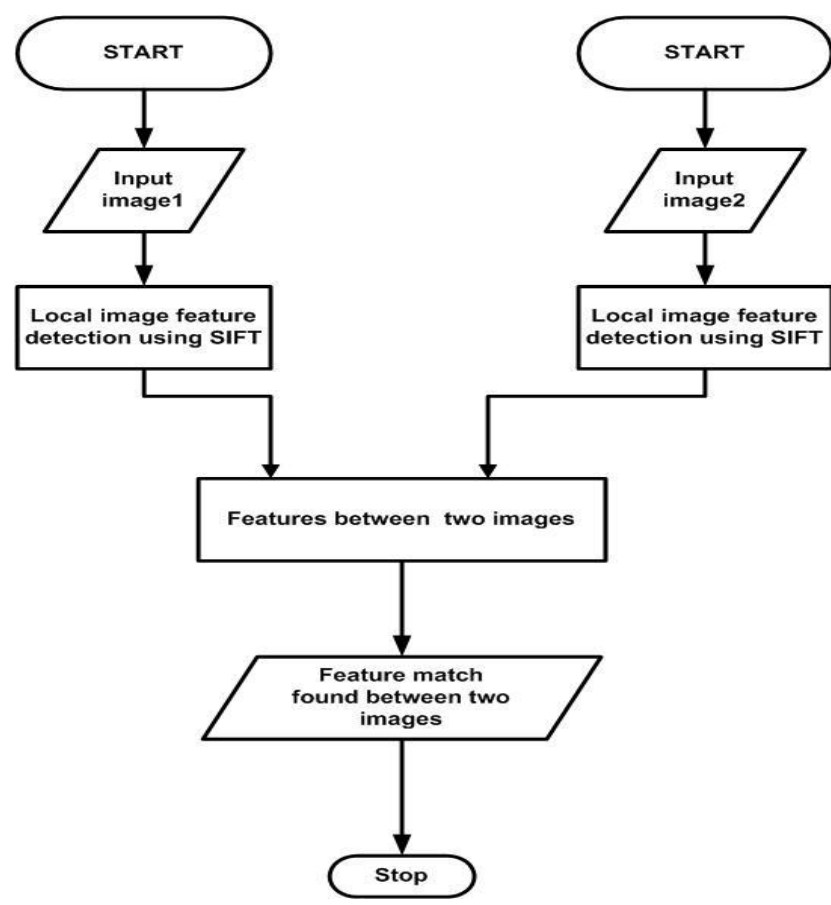

Figure 3.2: Flowchart for SIFT based match between two images

\subsection{Homography Using Ransac Algorithm}

RANSAC stands for "RANdom SAmple Consensus". RANSAC is a robust method to estimate parameters of a mathematical model from a set of observed data which contains outliers by an repeatative process. This method generates a reasonable result only with a certain probability. With this probability the repeatation increase is allowed. The algorithm was first developed by Fischler and Bolles at SRI international in 1981. Even though the method is old its robustness has made it useful in many applications. The algorithm is performed for a set of points detected or precomputed. In our implementation RANSAC is applied for the points set for the image which are obtained using SIFT feature detection algorithm.

The steps for the RANSAC estimation is as follows:

1. Obtain $\mathrm{n}$ points (in this case this value is from the matched features from two images).

2. Initialize the number of points, iterations, threshold inlier.

3. Compute for best fit using random points ( fit using 2 random points)

4. Count the inliers, if more than threshold Inlier, refit else iterate

5. Choose the coefficient with the most inliers

\section{RESULT ANALYSIS}

Figure 4.1(a) and 4.1(b) are the two overlapped input images. The number keypoints for Figure 4.1(a) is found to be 1400 and for Figure 4.1(b) is 995. The similar features found between Figure 4.1(a)and Figure 4.1(b) is 259. The SIFT match between two images is as shown in Figure 4.2. The transformation is obtained for image B with respect to the image A. This transformation of image is as shown in Figure 4.3(a). The transformation obtained is based on the homography matrix which is as given in Table 4.1. The wrapped image with redundancy removed using the implementation method described so far is as shown in Figure4.3(b).

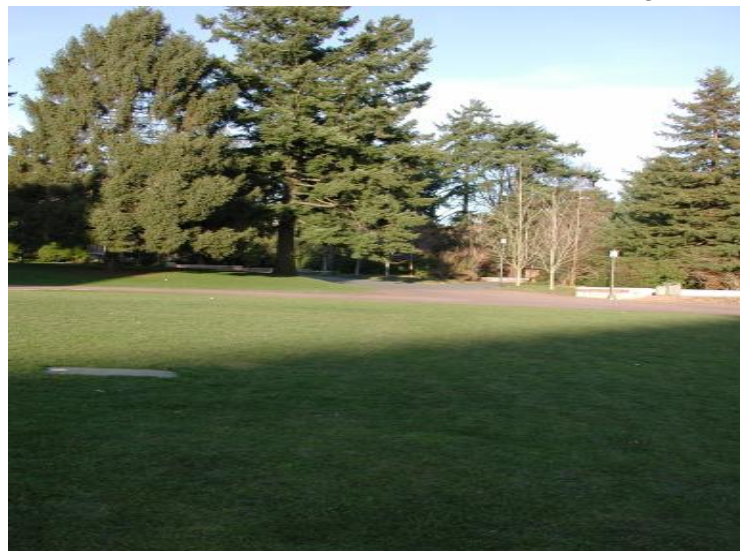

Figure 4.1:(a) Image A

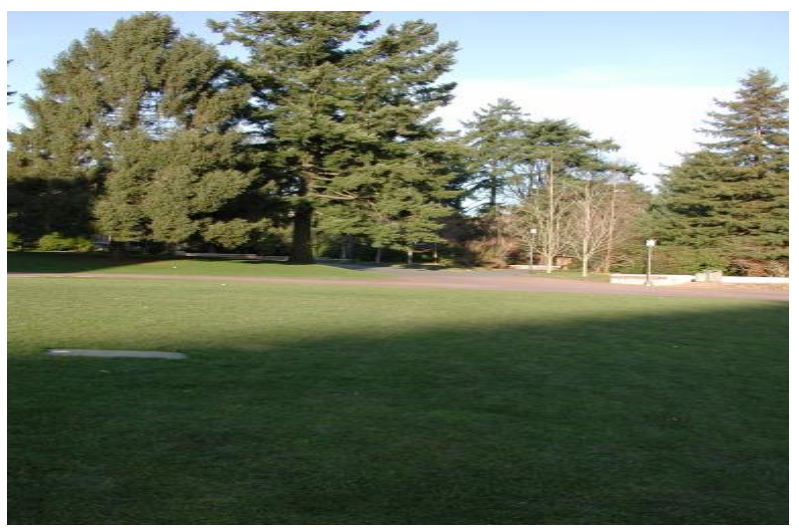

Figure4.1 (b) Image B

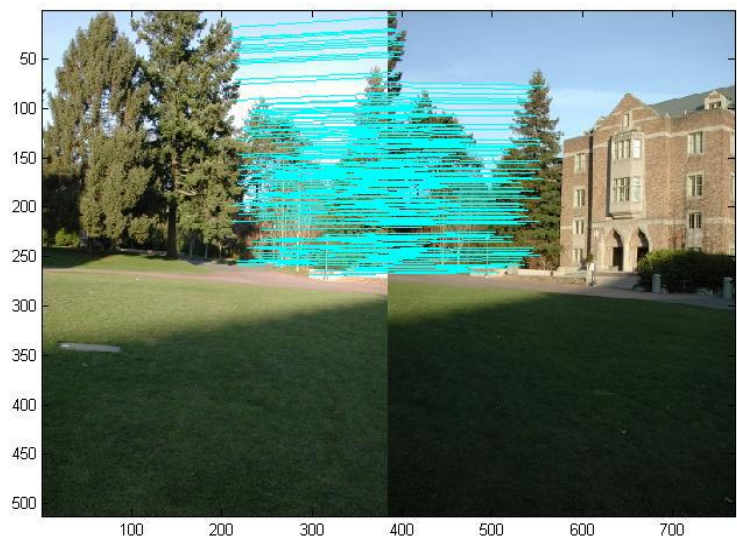

Figure 4.2: SIFT feature matched between two images

Table 4.1: Simulation results for two overlapped input images.

\begin{tabular}{|c|c|}
\hline FIGURE & $\begin{array}{l}\text { RESULT FROM THE } \\
\text { SIMULATION }\end{array}$ \\
\hline Keypoints for image A & 1400 \\
\hline Keypoints for image B & 995 \\
\hline $\begin{array}{l}\text { Matched keypoints between } \\
\text { two images }\end{array}$ & 259 \\
\hline $\begin{array}{c}\text { Homography matrix } \\
\text { estimation between two } \\
\text { images, } \mathrm{H}\end{array}$ & 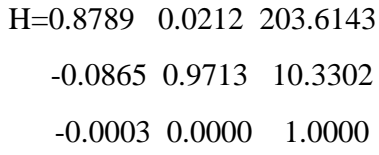 \\
\hline
\end{tabular}




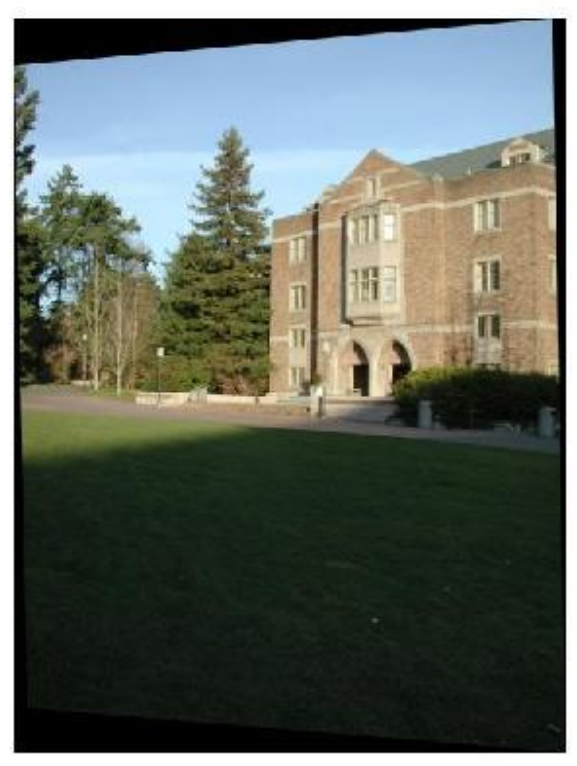

(a)

Image A transformed with respect to Image B

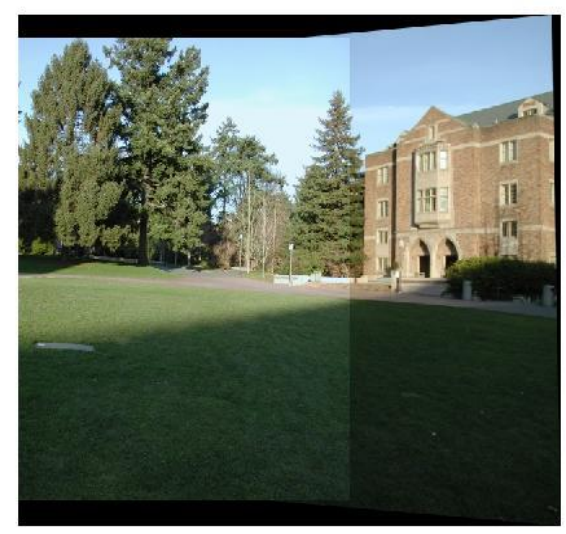

(b) Mosaiced output image

Figure 4.3: Transformation and Mosaic output.

\section{CONCLUSION}

The correlation coefficient measures the amount of overlapped regions between all $\mathrm{N}$ images. The results provide a $[\mathrm{NXN}]$ correlation matrix for $\mathrm{N}$ images. Joint entropy is used to measure the amount of information obtained when two images are combined. For merging process in the case of multiple images, SIFT based image mosaic technique is implemented. In total it can be concluded that Entropy Correlation Co-efficient model for redundancy removal in WMSN for visual data is achieved.

\section{REFERENCES}

[1] Dai and Akyildiz, "A spatial connection model for visual data in remote sight and sound sensor systems," IEEE
International Journal of Computer Applications (0975 - 8887)

Volume 148 - No.2, August 2016

TRANSACTIONS ON MULTIMEDIA, vol. 11, Oct 2009.

[2] Dai and Akyildiz, "A spatial connection based picture pressure structure for remote media sensor systems," IEEE TRANSACTIONS ON MULTIMEDIA, vol. 13, April 2011.

[3] Lowe, "Particular picture highlights from scale-invariant keypoints," International Journal of Computer Vision, vol. 60 .

[4] Ma and Liu, "Relationship based video preparing in video sensor systems," IEEE Int. Conf.Wireless Networks,Communications and Mobile Computing, vol. 2.

[5] D. Devarajan, Z. Cheng, and R. J. Radke, "Aligning circulated camera systems," Proc. IEEE, vol. 96, Oct 2008.

[6] Wiegand, Sullivan, Bjntegaard, and A. Luthra, "Diagram of the h.264/ave video coding standard," IEEE Trans. Circuits Syst. Video Technol., vol. 13, July 2003.

[7] $\mathrm{Wu}$ and Abouzeid, "Vitality proficient circulated picture pressure in asset compelled multihop remote systems," ELSEVIER, Computer Communications, vol. 28, Sep 2005.

[8] Wang, Bovik, Sheik, and Simoncelli, "Picture quality evaluation: From blunder perceivability to basic closeness," IEEE Trans. Picture Process., vol. 13, April 2004.

[9] R. Puri, A. Majumdar, and K. Ramchandran, "Crystal: A video coding worldview with movement estimation at the decoder," IEEE Trans. Picture Process., vol. 16, Oct 2007.

[10] Pluim, . Maintz, and Viergever, "Common data based enlistment of restorative pictures: A review," IEEE Trans.Med. Imag., vol. 22, no. 8, 2010.

[11] Studholme,. Slope, and Hawkes, "A cover invariant entropy measure of $3 \mathrm{~d}$ restorative picture arrangement," Pattern Recognition, vol. 32, no. 1.

[12] Jain, Murty, and P. J. Flynn, "Information grouping: An audit," ACM Comput. Surv., Sep 1999.

[13] A. Fathimaa, R.Karthikb, and V.Vaidehic, "Picture sewing with consolidated minute invariants and filter highlights," Pattern Recognition, vol. 32, no. 1.

[14] C. E. Shannon, "A numerical hypothesis of correspondence," The Mathematical Theory of Communication, 1949.

[15] R. Szeliski, "Image alignment and stitching," in JA Tutorial1, Microsoft Re-search, Microsoft Corporation, (http://www.research.microsoft.com), 204. 